\title{
Two-Photon Imaging during Prolonged Middle Cerebral Artery Occlusion in Mice Reveals Recovery of Dendritic Structure after Reperfusion
}

\author{
Ping $\operatorname{Li}^{1}$ and Timothy H. Murphy ${ }^{1,2,3}$ \\ ${ }^{1}$ Kinsmen Laboratory, Department of Psychiatry, ${ }^{2}$ Brain Research Center, and ${ }^{3}$ Department of Cellular and Physiological Sciences, University of British \\ Columbia, Vancouver, British Columbia, Canada V6T 1 Z3
}

\begin{abstract}
Filament occlusion of the middle cerebral artery (MCA) is a well accepted animal model of focal ischemia. Advantages of the model are relatively long occlusion times and a large penumbra region that simulates aspects of human stroke. Here, we use two-photon and confocal microscopy in combination with regional measurement of blood flow using laser speckle to assess the spatial relationship between the borders of the MCA ischemic territory and loss of dendrite structure, as well as the effect of reperfusion on dendritic damage in adult YFP (yellow fluorescent protein) and GFP (green fluorescent protein) C57BL/6 transgenic mice with fluorescent (predominantly layer 5) neurons. By examining the spatial extent of dendritic damage, we determined that $60 \mathrm{~min}$ of MCA occlusion produced a core with severe structural damage that did not recover after reperfusion (begins $\sim 3.8 \mathrm{~mm}$ lateral to midline), a reversibly damaged area up to 0.6 $\mathrm{mm}$ medial to the core that recovered after reperfusion (penumbra), and a relatively structurally intact area $(\sim 1 \mathrm{~mm}$ wide; medial penumbra) with hypoperfusion. Loss of structure was preceded by a single ischemic depolarization $122.1 \pm 10.2 \mathrm{~s}$ after occlusion onset. Reperfusion of animals after $60 \mathrm{~min}$ of ischemia was not associated with exacerbation of damage (reperfusion injury) and resulted in a significant restoration of blebbed dendritic structure, but only within $\sim 0.6 \mathrm{~mm}$ lateral of the dendritic damage structural border. In summary, we find that recovery of dendritic structure can occur after reperfusion after even $60 \mathrm{~min}$ of ischemia, but is likely restricted to a relatively small penumbra region with partial blood flow or oxygenation.
\end{abstract}

Key words: stroke; imaging; dendritic spine; blood flow; ischemia; dendrite

\section{Introduction}

The large majority (80\%) (Durukan and Tatlisumak, 2007) of human strokes are ischemic and involve the occlusion of a major artery, commonly the middle cerebral artery (MCA). This event can be modeled in rodents by advancing a nylon suture up the common carotid artery (CCA) to block the MCA and withdrawing it after 60-90 min to induce reperfusion (Longa et al., 1989; Kawamura et al., 1991). The model has been applied in rodent studies of neuroprotection, as well as neuroplasticity after stroke (Chen et al., 1992, 1999; Iijima et al., 1992; Bethel et al., 1997; Ma et al., 1998), and extended to transgenic mice to examine targets that modify damage (Kamii et al., 1994; Hara et al., 1996). This approach parallels human ischemic stroke by permitting occlusion over hours and supporting an ischemic penumbra (area with partial vascular flow) in which the beneficial effects of neuropro-

\footnotetext{
Received Aug. 6, 2008; revised Sept. 24, 2008; accepted Sept. 29, 2008.

This work was supported by operating grants from Canadian Institutes of Health Research (MOP49586), the Heart and Stroke Foundation of British Columbia and Yukon, the Canadian Stroke Network, and the University of British Columbia In Vivo Imaging Centre (T.H.M.). We thank Pumin Wang for outstanding animal surgeries, Richard Liu for assistance in preliminary experiments, and Heidi Erb, Xiaoyan Jiang, and Jessica Macleod for help with data analysis. Correspondence should be addressed to Dr. Timothy H. Murphy, Department of Psychiatry, University of British Columbia, 4N1-2255 Wesbrook Mall, Vancouver, British Columbia, Canada V6T 1Z3. E-mail: thmurphy@interchange.ubc.ca. P. Li's present address: Jacobs Neurological Institute, 100 High Street, Buffalo, NY 14203. D0I:10.1523/JNEUROSCI.3724-08.2008

Copyright $\odot 2008$ Society for Neuroscience $\quad$ 0270-6474/08/2811970-10\$15.00/0
}

tectants and reperfusion are thought to occur (Hossmann, 2006). The wide application of this model stems from its ability to produce defined ischemic episodes and reperfusion in a controlled manner. Although the model is commonly used, it is not known how fine dendritic structure is affected during both occlusion and reperfusion in both the core and the penumbra.

Recent in vivo two-photon imaging experiments have focused on synapses as targets of acute ischemia (Zhang et al., 2005; Zhang and Murphy, 2007; Murphy et al., 2008). These studies indicate rapid swelling and beading of dendritic structure and a loss of spines within minutes of global ischemia coincident with a wave of ischemic depolarization (Murphy et al., 2008). Dendritic structure can be markedly disturbed during global ischemia, whereas reperfusion can lead to recovery of structure within tens of minutes (Murphy et al., 2008). Although the recovery of structure with reperfusion was remarkable, initial studies only used an average of $7 \mathrm{~min}$ of occlusion (Murphy et al., 2008), or used photothrombotic occlusion in which reperfusion was undefined (Zhang et al., 2005), thus limiting their power as models of human focal stroke that typically involve the occlusion of major arteries for hours. Although reperfusion permitted recovery of dendritic structure after short-term occlusion (Zhang et al., 2005; Murphy et al., 2008), it is conceivable that reperfusion may lead to exacerbation of injury (Aronowski et al., 1997; GürsoyOzdemir et al., 2004) when combined with longer periods of 
occlusion. Out of these limitations, several important questions arose: (1) if occlusion is maintained for hours, will dendritic structure recover during reperfusion; and (2) if structural recovery occurs, is it limited to penumbra regions near the stroke border, or does it include the ischemic core; (3) will the penumbra be subject to reperfusion damage after prolonged occlusion? Here, we adapt the mouse model of MCA occlusion (MCAO) for use with two-photon imaging to address these questions. We define the MCA territory within each animal and show that dendritic structure within this area is markedly blebbed during $1 \mathrm{~h}$ of MCAO. After reperfusion, dendrites within the ischemic penumbra can recover, but recovery is limited to a small region and does not extend to the ischemic core.

\section{Materials and Methods}

Transgenic mice. We have studied a total of 22 adult, male, 9-15 weeks of age, and 23-31 g YFP-H and GFP-M transgenic mice (Feng et al., 2000). All experiments used urethane anesthesia as in the studies by Zhang et al. (2005), Zhang and Murphy (2007), and Murphy et al. (2008). C57BL/6 yellow fluorescent protein (YFP)- and green fluorescent protein (GFP)expressing transgenic mice ( $\mathrm{H}$ and $\mathrm{M}$ lines) (Feng et al., 2000) were bred at the University of British Columbia animal facilities. Briefly, anesthesia was induced with urethane $(0.12 \% \mathrm{w} / \mathrm{w})$ and body temperature was maintained at $37 \pm 0.5^{\circ} \mathrm{C}$ using a heating pad and feedback regulation from a rectal temperature probe. Hydration was maintained by intraperitoneal injection of saline $(200-300 \mu \mathrm{l})$ with $20 \mathrm{~mm}$ glucose at $1-2 \mathrm{~h}$ intervals. The experimental protocols were approved by the University of British Columbia animal care committee and consistent with Canadian Council on Animal Care and Use guidelines.

Surgical procedures and imaging. The surgical procedures for preparing a cranial window and the in vivo two-photon imaging methods have been described previously (Zhang et al., 2005; Zhang and Murphy, 2007; Murphy et al., 2008). Briefly, a craniotomy was performed over the right somatosensory cortex from 1 to $4.5 \mathrm{~mm}$ lateral to midline. Animals were fitted into a custom-made head hold. Two-photon excitation was performed with a Coherent Mira 900 Ti-sapphire laser pumped by a $5 \mathrm{~W}$ Verdi laser and tuned to $900 \mathrm{~nm}$ to excite YFP or GFP. The cortex was covered with $1.3 \%$ low-melt agarose (at $37-38^{\circ} \mathrm{C}$; type 3-A; Sigma-Aldrich; A9793) dissolved in HEPES-buffered artificial CSF and sealed with a glass coverslip (no. 1) as in the study by Zhang et al. (2005). Images were acquired by custom software (Igor) and by using an Olympus IRLUMPlanFl water-immersion objective ( $40 \times ; 0.8$ numerical aperture). The images were taken with a pixel size of $0.3 \mu \mathrm{m}$, typically over a $1.2-$ $1.8 \times 0.3 \mathrm{~mm}$ band of tissue that spanned over both the expected MCA and anterior cerebral artery (ACA) territories. Each image was the average of two frames taken over $3.7 \mathrm{~s}$. To image blood flow, blood plasma was labeled through a tail vein injection of a $0.2 \mathrm{ml}$ bolus of $5 \%(\mathrm{w} / \mathrm{v})$ Texas Red dextran (70 kDa) (Invitrogen; D-1830) in PBS (Kleinfeld et al., 1998; Zhang et al., 2005; Nishimura et al., 2006; Schaffer et al., 2006).

Tracheostomy was performed on all animals before producing the stroke to alleviate breathing problems that may have been confounded by manipulation of the tissue within the neck region or prolonged anesthesia. A ventral midline neck incision was made. The thyroid and associated muscle and connective tissue were carefully dissected away from the trachea. A PE-90 catheter was inserted into the trachea and secured with VetBond.

For the MCAO model of stroke, ischemia-reperfusion was induced using a modified version of the intraluminal suture method as described previously in rats (Longa et al., 1989). Briefly, laser Doppler flow measurements (Perimed PeriFlux PF3) were performed through the skull at the right temporal fossa (see Fig. $2 \mathrm{~A}$ ). After the tracheostomy, the right CCA was carefully dissected away from the vagus nerve. The ipsilateral external carotid artery (ECA) was ligated. A $2 \mathrm{~cm}$ 6-0 nylon suture (Ethilon; Ethicon) (the diameters of the tips, which were blunted with a microforge, were $\sim 165 \mu \mathrm{m}$ ) was inserted through the CCA and pushed into the internal carotid artery (ICA) until the laser Doppler recording showed a sharp blood flow decrease, leaving $\sim 10-11 \mathrm{~mm}$ of the suture remaining outside the ECA/ICA bifurcation. The suture was clamped in place with a microaneurysm clip (Harvard Apparatus), and the animal was then put back to the stage for imaging. After $\sim 60 \mathrm{~min}$ of ischemia, the suture was retracted. Although both the right CCA and ECA were permanently ligated, blood flowing from the contralateral CCA, and possibly ipsilateral posterior circulation, could still pass through the MCA allowing $\sim 70 \%$ reperfusion. Animals were included in the study based on two criteria: (1) they must have a sharp drop of blood supply to the temporal cortex from the laser Doppler recording (to $<40 \%$ of baseline blood flow), and (2) they must have a decrease in number of flowing blood vessels on the side of the craniotomy that contained the MCA territory as assessed by laser speckle imaging. Occlusion was also confirmed by in vivo two-photon imaging of blood flow as previously described (Zhang et al., 2005).

After reperfusion, the animals were followed with two-photon microscopy for $2-3 \mathrm{~h}$. Then they were transcardially perfused with PBS, followed by $4 \%$ paraformaldehyde in PBS. The brains were removed. Coronal sections were cut at $100 \mu \mathrm{m}$ thick on a vibratome. For histological study, images were captured with use of either a Zeiss Axiophot light microscope coupled to a 12-bit CCD camera (Retiga; QImaging) or an Olympus Fluoview 1000 confocal microscope.

The procedures for laser speckle imaging have been described previously (Dunn et al., 2001; Sigler et al., 2008). Briefly, the cortical surface was illuminated by a $784 \mathrm{~nm}, 32 \mathrm{~mW}$ StockerYale SNF-XXX-785S-35 laser (StockerYale) with an Edmund Optics anamorphic beam expander T47274 (Edmund Optics), and 50-150 images at $30 \mathrm{~Hz}$ using 10-15 ms exposure were collected with a Dalsa M60 Pantera camera (Dalsa).

Electroencephalogram recording. For DC potential recording, we used a DAM 50 amplifier (WPI) using chlorided Teflon-coated silver wire $(0.125 \mathrm{~mm}$; WPI) placed on the surface of the cortex suspended within the overlying agarose usually within the lateral aspect of the craniotomy within 1-2 $\mathrm{mm}$ of the dendritic structural border. Reference electrodes were placed over the animal's back between the scapula and on the paw (filter set at $0.1-1000 \mathrm{~Hz}$ ). Additional off-line filtering of $10-20 \mathrm{~Hz}$ low pass was also performed routinely to better resolve slow ischemic depolarization. Electroencephalogram (EEG) data were collected using Clampex 8 or Axoscope 10.2 and analyzed using Clampfit 8 or 9 (Molecular Devices).

In addition to measuring the heart rate electrophysiologically (in most animals), we also assessed physiological parameters using a Starr Life Sciences Mouse-Ox pulse oximeter mounted on the tail. Assessment of blood oxygen saturation (typically $>90 \%)$ and heart rate $(400-600$ beats $/ \mathrm{min}$ ) in a subset of animals indicated that, under the conditions we used for imaging, physiological parameters were relatively constant over the course of our experiments. Consistent with the MCAO being associated with focal ischemia, we did not observe suppression of the heart rate during the occlusion period. All animals were under urethane anesthesia and were freely breathing air during all imaging experiments either through their nose (before the stroke) or a PE-90 tube within the tracheotomy after stroke induction.

Image analysis. Image analysis was performed using ImageJ software (http://rsb.info.nih.gov/ij/). To reduce photon and photomultiplier tube noise, a median filter (radius, 1) was applied to all images. Dendritic blebbing measurements were done as previously described (Murphy et al., 2008). To reduce bias, images of dendritic panels oriented medial to lateral were cut into $250 \times 1024$ pixel-sized sections, given random designations, and scored by persons who lacked information regarding the treatments/time point used or medial-to-lateral orientation. Briefly, to assess dendritic blebbing in two-photon images, we estimated the fraction of blebbed dendrites by scoring $20 \mu \mathrm{m}$ dendritic segments observed in maximal intensity projections as well as the three-dimensional stack as normal or blebbed for time points taken before, during, or after MCAO. Counting the segments was aided by the use of grid with $20 \mu \mathrm{m}$ squares placed in ImageJ. Blebbed dendrites have regular swellings and have a "beads on string" appearance as described previously by us (Zhang et al., 2005) and investigators working in vitro and with histology (Hori and Carpenter, 1994; Park et al., 1996; Andrew et al., 2007). We scored dendrites as having blebs if they exhibited regularly spaced and rounded herniations that were punctuated by interbleb regions with lower or, in 
some cases, nearly undetectable fluorescence. Blebbed regions were of larger diameter than their parent dendrite. These rounded structures were distinct from axonal boutons and passing dendrites cut in cross section that were observed in all animals before stroke and were checked by examining three-dimensional stacks in addition to $Z$-projections.

For speckle imaging analysis, we randomly selected three to five arteries that were within the MCA territory and did not have clear collateral connections with the ACA from each animal. We quantified blood flow velocity change from the same large vessels by examining changes in speckle contrast signal before and after advancement of the suture, and after withdrawal of the suture. Results were expressed as a percentage of pre-CCA occlusion speckle contrast (which was used as 100\%). In this analysis, we assume that the velocity of blood flow is proportional to the inverse of the speckle contrast as shown by Suzuki et al. (1991) and scaled over a range corresponding to maximal and minimal flow for each animal. We also analyzed the line profile of speckle contrast made by averaging vertical (rostral-caudal) pixels for each $1024 \times$ 100 pixel area. The site along the line profile (medial-lateral dimension) in which the $\mathrm{MCAO}$-induced change in speckle contrast value was altered by $50 \%$ (minimal - maximal speckle contrast $/ 2^{\star} 100$ ) was considered the border between ACA and MCA territories. To avoid variability caused by the intermittent nature of large surface vessels, these distance estimates were made from regions lacking large surface vessels, over capillary and small vesselrich areas that also contribute to speckle signals (Dunn et al., 2001).

For statistical analyses of three or more groups, an ANOVA was used with an $\alpha$ value for significance of 0.05; in cases in which multiple post hoc comparisons were made, Bonferroni's multiple-comparison test was used. Comparison of two groups was made by $t$ test. Data are expressed as mean \pm SEM, unless otherwise stated.

\section{Results}

Blood flow and structural change during MCAO

The general experimental scheme for assessment of blood flow and dendritic structure before, during, and after MCAO is outlined in Figure 1. In urethaneanesthetized adult mice, we assessed blood flow during MCAO by attaching a laser Doppler probe to the temporal aspect of the skull, which permitted us to monitor regional blood flow in the MCA territory during both the occlusion and reperfusion processes (Fig. 2A). We found it necessary to perform continuous laser Doppler readings during surgical procedures to optimize suture placement for induction of ischemia. In addition, continuous monitoring confirmed that occlusion was stable while inverting the animal from ventral side up (that was required to access the carotid arteries) to dorsal side up for two-photon imaging. Importantly, the laser Doppler probe could be positioned on the lateral cortex without disturbing the ability to perform two-photon microscopy within more medial regions in which the

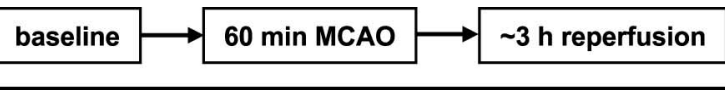

assess blood flow, structure, and electrical events.

Figure 1. Experimental setup and timeline for focal stroke imaging. The timeline of a MCAO reperfusion experiment is shown. Baseline laser speckle images and two-photon images of dendritic structures and blood vessels were taken. The animal was moved to a surgery area and a nylon suture was advanced to block the MCA inducing stroke. With the suture blocking the MCA, the animal was returned to the microscope, and laser speckle and two-photon images were taken from the same areas. After $\sim 60$ scope, and laser speckle and two-photon imaging were continued for $\sim 2.5 \mathrm{~h}$ (after the start of reperfusion) at which time the
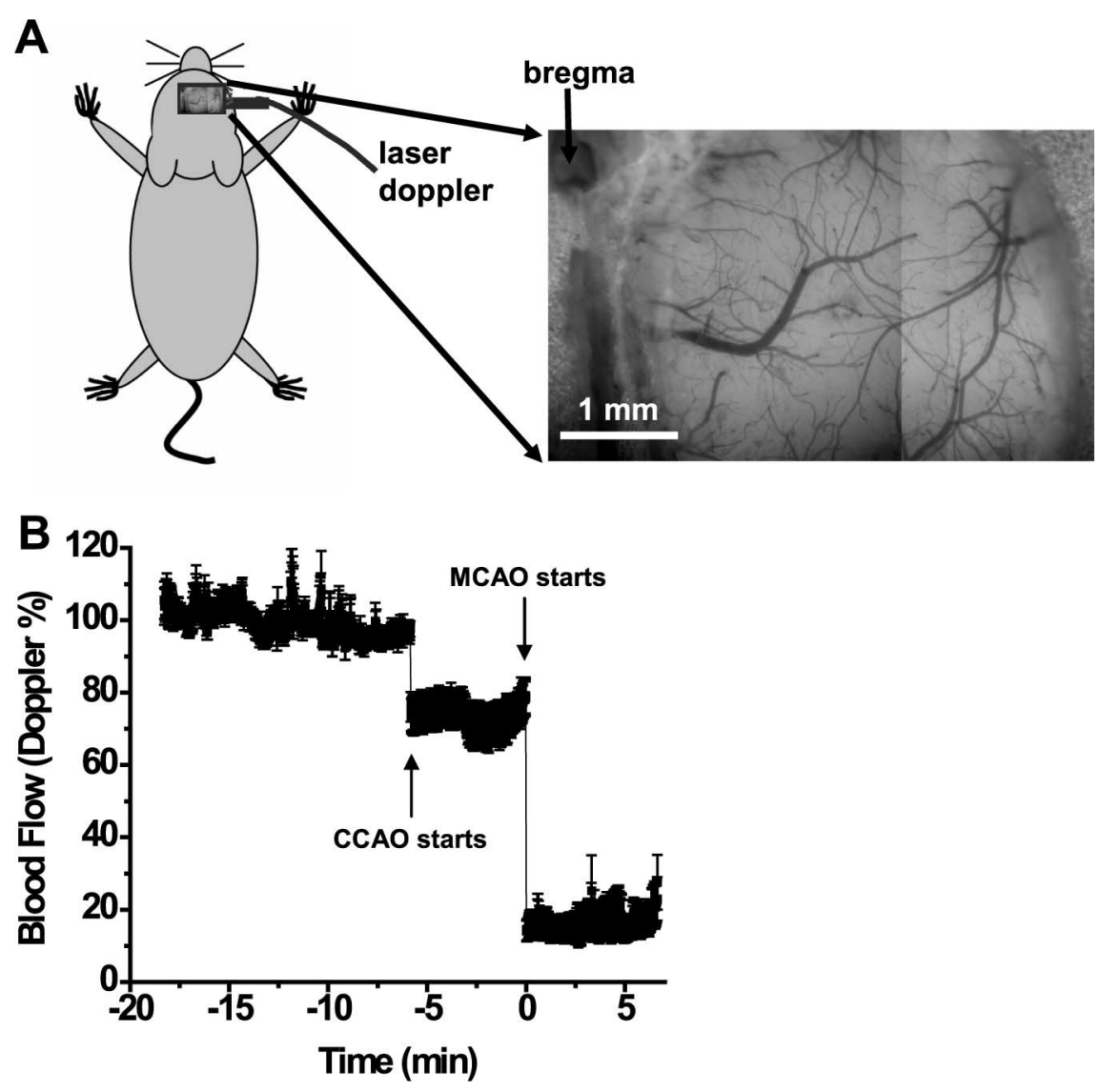

Figure 2. Regional assessment of cortical blood flow using laser Doppler. $\boldsymbol{A}$, Diagram showing location of the laser Doppler probe on the lateral skull surface and cranial window location over the right hemisphere just posterior of the bregma point. $\boldsymbol{B}$, Group data from 22 animals (mean \pm SEM) showing relative changes in laser Doppler signal measured from the lateral aspect of the skull over the MCA core region. After unilateral CCAO (necessary to insert the suture), an $\sim 20 \%$ reduction in blood flow was observed. An additional large reduction in blood flow to $10 \%$ of normal flow ( $~ 90 \%$ in total) occurs within the MCA territory when the suture was advanced to the MCA. The recording ends at $\sim 6$ min after the onset of occlusion because the animal needs to be moved to the two-photon microscope stage and laser Doppler recording was no longer possible at this point.

stroke penumbra was present. The first step in the MCAO procedure is ligating the ipsilateral CCA, permitting the insertion of a nylon suture that is advanced to occlude the MCA. Ligation of the CCA resulted in the blood flow being reduced to $74 \pm 4 \%$ of baseline levels (Fig. 2 B). Advancing the suture further decreased the Doppler blood flow signal to $16 \pm 4 \%$ (of baseline levels) when the suture tip occluded the MCA (Fig. $2 B$ ).

To assess regional blood flow within the area in which twophoton imaging was performed, we used laser speckle imaging in which local variance in laser speckle pattern provided a measure of blood flow velocity (Suzuki et al., 1991). The laser speckle images are presented as speckle contrast (local SD/mean) with darker tones indicating higher velocity blood flow (see Materials 


\section{A Laser speckle images}

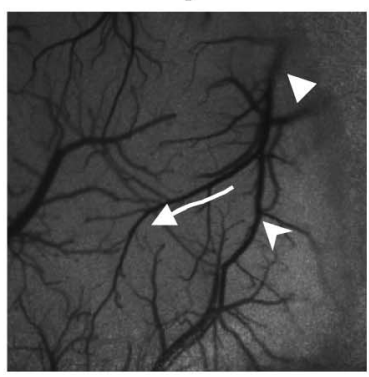

Pre-CCAO

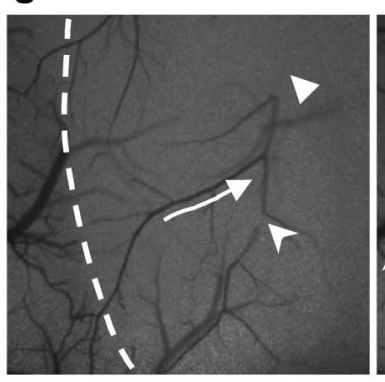

MCAO

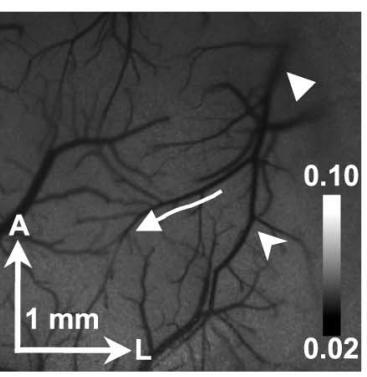

Reperfusion
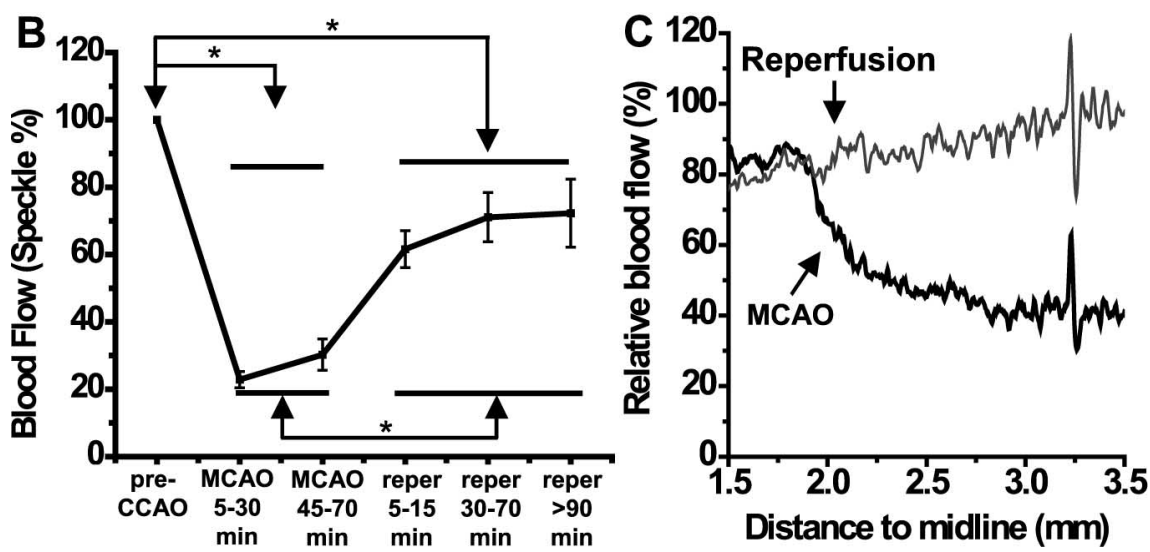

Figure 3. Regional assessment of cortical blood flow using laser speckle imaging. $\boldsymbol{A}$, Laser speckle images showing surface blood flow with high-velocity blood flow presented as darker gray tones. The images were calculated as described in Materials and Methods and reflect local SD of speckle patterns divided by the mean gray value. The calibration bar indicates the ratio of speckle SD to the mean reflectance in a range of $0.02-0.10$. Occlusion of the right MCA results in a large blood flow deficit, which is greatest on the right portion of the image shown (more lateral cortical regions). The image marked pre-CCAO shows blood flow before unilateral occlusion of the CCA that was necessary to insert the suture inducing the ischemia. The middle panel marked MCAO was taken 19 min after suture occlusion of the MCA. The right panel marked reperfusion showed the speckle image taken after reperfusion (49 min of reperfusion). The white arrowheads indicate the blood vessel flowing before MCAO and after reperfusion, and stalled during MCA0. The white arrows show the direction of blood flow indicated by two-photon microscopy study from the same area. The direction of flow reversed during MCA0, suggesting that the blood flow was derived from the ACA instead of MCA system. $\boldsymbol{B}$, Quantification of local blood flow (mean \pm SEM) using laser speckle within the most lateral of the imaging window used for two-photon microscopy. Data shown are the average from 16 separate animals. In this case, laser speckle values were inverted and scaled over a range from maximal to minimal blood flow (as described in Materials and Methods) to better illustrate changes. A significant difference $\left({ }^{*} p<0.05\right)$ was observed between preocclusion and during occlusion, preocclusion and after reperfusion, and during occlusion and after reperfusion (by one-way ANOVA). $C$, Line profiles of laser speckle signal from $1024 \times 200$ pixels ( $300 \times 60 \mu \mathrm{m}$, averaged in the vertical dimension) from the center of the three images in $\boldsymbol{A}$. Data are expressed as percentage of prestroke speckle contrast (see Materials and Methods) so that greater values correspond to greater blood flow. During MCAO, there was less blood flow to the more lateral region containing the MCA territory. The blood supply recovered to almost normal values after reperfusion.

and Methods) (Fig. 3A). Toward the medial extent of the MCA territory, a blood flow border $(2.1 \pm 0.1 \mathrm{~mm}$ lateral from the midline; $n=17$ animals) was apparent between the more lateral MCA and medial ACA territories. Moving more lateral (away from the border) flow within the surface vessel network was strongly attenuated by MCAO with some vessels showing little or no laser speckle signal (Fig. 3A). Toward the midline, the blood supply was less sensitive to MCAO and was apparently from the more medial ACA system.

To graphically show laser speckle data in a more intuitive manner, we presented the inverse of speckle contrast (scaled over a range corresponding to maximal and minimal flow) (see Materials and Methods), so that higher numbers correspond to higher velocities of blood flow (Fig. 3B). On average, 1/speckle contrast (proportional to blood flow velocity) (Briers and Fercher, 1982) dropped to $24 \pm 3 \%$ of baseline values during MCAO (Fig. 3B).
Baseline blood flow was defined from speckle images taken before unilateral CCA occlusion (CCAO). Importantly, the changes in laser speckle signal were similar in magnitude to the laser Doppler measurements (Fig. $2 B$ ), assuming some expected variance because the Doppler probe was placed over the core of the MCA territory, whereas the speckle images were taken from a more medial area. Changes in speckle contrast were also observed after MCAO in capillary-rich areas (Yuan et al., 2005) lacking large surface vessels (background signal present in images) (Fig. $3 A$ ) (quantified in Fig. 3C).

In all animals studied, there was apparent overlap between the MCA and ACA territories leading a relatively wide blood flow transition zone (from fully flowing to severely depressed) during MCAO. Although blood flow was generally depressed (within the presumed MCA territory), we often found spared arteries with little or no block of blood flow by MCAO (Fig. 3A). By examining two-photon images that can define the direction of blood flow (Kleinfeld et al., 1998; Zhang et al., 2005), we found reversal in blood flow direction (data not shown), which suggested that these arteries were now supplied by the more medial ACA system instead of MCA system (Fig. 3A, long arrow).

As another means of assessing the effect of MCA occlusion, we included EEG surface electrodes (in some animals) within the craniotomy in which two-photon imaging was performed as previously described (Murphy et al., 2008). Within seconds of occlusion, we observed a strong reduction in spontaneous EEG power consistent with ischemia blocking ongoing synaptic activity (Fig. 4A). Appearing at $122 \pm 10 \mathrm{~s}(n=6$ animals $)$ after MCAO onset, we observed a single ischemic DC depolarization (Fig. 4B), which was not significantly different in latency from the DC shift after global ischemia induced by bilateral CCAO (110 $\pm 11 \mathrm{~s} ; n=10$ animals) (Murphy et al., 2008). In the global ischemia model, changes in dendritic structure were initiated at the point of ischemic depolarization. Although we could not begin imaging within the first $5 \mathrm{~min}$ of MCAO, these findings suggest that there would be similar mechanisms used in the two models. Namely, ischemic depolarization would underlie the early dendritic structural damage during both focal and global ischemia. The amplitude of the DC potential shift after MCAO was smaller than that observed after global ischemia ( $9 \pm 1 \mathrm{mV}, n=7$, for MCAO; $12 \pm 1 \mathrm{mV}, n=8$, for $\mathrm{CCAO} ; p<0.05)$. Conceivably, a smaller amplitude depolarization in the focal model could be related to the surface EEG electrode sampling from some territories that were not completely ischemic. Using surface stimulation, we estimate that the EEG electrodes used will measure signals from areas up to $1 \mathrm{~mm}$ away with $<50 \%$ signal attenuation (our unpublished data). We can- 
not rule out that ongoing small periinfarct ischemic depolarizations (Hossmann, 1996, 2006) that could aggravate dendritic damage may go undetected because their measurement may be hampered by use of a large electrode.

\section{Effects of MCAO on dendritic fine structure}

After confirming occlusion within the MCA territory, we examined the potential effects of MCAO on dendritic structure of layer 5 neurons expressing YFP or GFP, and the relationship between the structure and local changes in capillary blood flow. Most neurons have their cell bodies in layer 5, although some lateral neurons that are not typically imaged are from layer II. Structure was assessed using two-photon imaging of apical dendritic tufts found within the first $100 \mu \mathrm{m}$ of cortex in layer 1. Imaging was initiated from the most lateral regions of the craniotomy, which are within the expected MCA territory. We observed a clear blebbing of apical dendrites (compared with preischemia images) when images were acquired on the most lateral side of the imaging window (closest to the MCAO core, typically 3.5-4.0 $\mathrm{mm}$ lateral from midline). We then made image stacks moving closer to the midline at $\sim 250 \mu \mathrm{m}$ intervals. These image stacks indicated a relatively sharp dendritic structural transition from blebbed to relatively intact at $3.1 \pm 0.1 \mathrm{~mm}$ lateral to midline ( $n=9$ animals) (Fig. 5A). For close-up images of dendritic structure and local blood flow, see Figure 5, $B$ and $C$. The structural border was defined as the area with the sharpest change in the percentage of the beaded dendrites. For animals $(n=4)$ having both a craniotomy and unilateral CCAO (only reduces blood flow by $\sim 25 \%$ ), we did not observe dendritic beading even after $5 \mathrm{~h}$ of unilateral CCAO (Zhang and Murphy, 2007). The incidence of beaded dendrites in MCAO animals was reduced from $65 \pm 4 \%$ to $33 \pm 4 \%$ ( $n=8$ animals) crossing the structural border and moving to the midline toward areas with greater blood flow (Fig. 5D). Because of the requirement to sample relatively large areas, and to use animals with a high density of fluorescently labeled neurons (to ensure sampling of all affected areas), we were unable to quantify the loss of individual dendritic spines and relied on measures of dendrite blebbing that were previously well correlated with spine loss (Murphy et al., 2008) (see Materials and Methods). Using a combined spatial analysis of blood flow (speckle) and blebbing, an area up to $1 \mathrm{~mm}$ medial to the structural border was hypoperfused (see examples in Figs. 3A, 5C, and the average of all animals in Fig. 7A), but contained relatively intact dendritic structure. Because of the time required to complete all stacks and laser speckle acquisition before reperfusion, we could not always image all the areas twice during occlusion. However, for the areas we managed to image twice, the incidence of dendritic beading increased over time ( $n=2$ animals, from 57 to $68 \%$ within $150 \mu \mathrm{m}$ lateral to the structural border over $20 \mathrm{~min}$ ). Moving toward the midline away from the MCA territory, we found mostly intact dendritic structure. As a control, we also quantified blebbing before MCAO (Fig. 5D) and found that it was low even at the edges of the craniotomy, suggesting that mechanical damage from surgery was minimal in the areas in which imaging was performed.

In some cases, structural damage borders were irregular, possibly because of patchy support by local blood flow, or through spatial heterogeneity in ACA and MCA territories (for example, see Fig. 3A). In the example shown in Figure 5, dendritic blebbing is most apparent on the lateral part of the image. Moving toward the midline, a patch of intact dendrites is present. However, moving even more toward the midline blebbing is again widespread suggesting patchy islands of intact tissue. To assess why this occurs, we show a laser speckle image from the area in which this patchy dendritic damage was observed. In this animal, an unblocked artery was indicated in the laser speckle image (Fig. 5C) and could account for some of the spared dendrites. Across the laser speckle image, a gradual darkening is apparent toward the medial portion of the image and is attributed to diffusion in microvessels (Dunn et al., 2001; Yuan et al., 2005; Sigler et al., 2008), indicating higher levels of local blood flow.

For microscopic assessment of blood flow, we labeled the vasculature with Texas Red dextran (Kleinfeld et al., 1998; Zhang et al., 2005; Nishimura et al., 2006; Schaffer et al., 2006). Given that we needed to image the dendrites over typically a $0.3 \times 1.5 \mathrm{~mm}$ area and that each stack takes $5-10 \mathrm{~min}$ to acquire, we did not have sufficient time within the $60 \mathrm{~min}$ of occlusion to quantify blood flow using line scan analysis of red blood cell velocity (Kleinfeld et al., 1998). Instead, we examined vessels for highvelocity red blood cell movement or stalling as previously described by us (Zhang and Murphy, 2007). We confirmed that the transition in dendritic structure was associated with local changes in capillary blood flow (supplemental Fig. 1, available at www. jneurosci.org as supplemental material).

\section{Blood flow and structural recovery after reperfusion}

Laser Doppler flow measurements were used to mark the initiation of reperfusion and provided feedback while the suture was retracted to unblock the MCA during the first few minutes of reperfusion. Because we needed to detach the laser Doppler probe during two-photon imaging, we relied on laser speckle imaging for confirmation of reperfusion, which could be performed with the head hold in place for two-photon imaging. Laser speckle imaging from regions of interest on presumed arteries demonstrated that we could reperfuse animals to $70 \pm 6 \%$ of their pre-CCAO blood flow (Fig. 3B). Although reperfusion seems incomplete, it is worth noting that unilateral CCAO is still in place, which reduces blood flow by $27 \pm 5 \%$ by laser Doppler assessment. Furthermore, laser speckle measurements of microcirculation (from areas without large vessels) indicated full reperfusion (Fig. 3C, for example) (for group data, see Fig. 7A). For animals in which MCAO and reperfusion could be confirmed $(n=8)$, we observed a reduction in dendritic blebbing when 

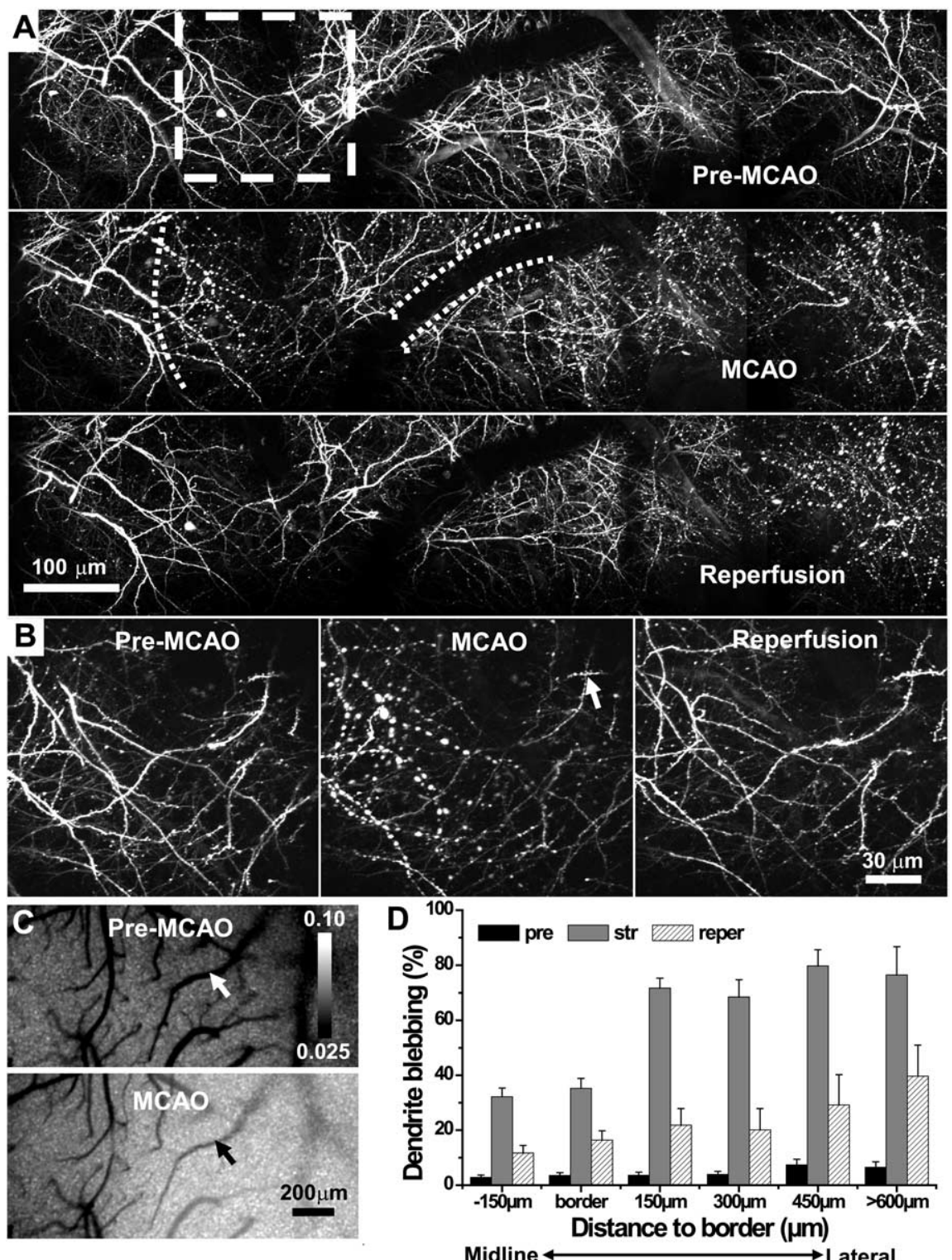

Figure 5. Two-photon imaging of local changes in blood flow and dendritic structure before, during, and after MCA0. $\boldsymbol{A}$, Two-photon projection images of dendritic structure before, during, and after MCA0. Each image was made from a projection derived from five $Z$ sections. They are oriented with the right side being more lateral, which is further into the MCA territory. The middle panels were taken during 26-64 min after MCA0. The dashed line in the left part shows the structural damage border. As one moves to the left away from the MCA core, the structure improves, indicating a transition zone or border. To the right toward the MCA core, degradation of the structure was observed. The dashed line over the vessel indicates an artery segment that was not completely blocked during MCA0 (see $\boldsymbol{C}$ ) and in which intact dendrites were observed. $\boldsymbol{B}$, Close images of dendritic structure within the dashed area in $\boldsymbol{A}$, indicating intact structure before MCAO In the middle panel, a projection image is shown 52 min after MCAO and extensive dendritic blebbing is observed. A small white arrow shows a region in which dendrites were relatively spared, possibly because of residual blood flow in the vessel indicated below in $\boldsymbol{C}$ (laser speckle images). In the right panel, the animal was reperfused and a significant recovery of dendritic structure was observed. $\boldsymbol{C}$, Laser speckle image displayed as speckle contrast before and $15 \mathrm{~min}$ after MCA0. During MCA0, a large reduction in blood flow is indicated by lighter gray level tones. An artery segment partially blocked during MCAO is indicated by white and black arrows in pre-MCAO and MCAO panels, respectively. The same vessel is indicated in $\boldsymbol{A}$ (during MCAO) in dendrite images by a white dashed line. D, Quantification of dendritic blebbing (mean \pm SEM) in eight animals before (pre), during MCAO stroke (str), and after reperfusion (reper). Data shown reflect the percentage of blebbed dendrites found at these various times at different distances from the apparent border of dendritic damage. The percentages of dendritic blebbing are significant different between prestroke control and during $\mathrm{MCA0}$, during $\mathrm{MCA0}$, and after reperfusion at all distances ( $p<0.05$, by two-way ANOVA). Between prestroke control and after reperfusion, it is significantly different at 450 and

$>600 \mu \mathrm{m}$ from the structural border, but not at other distances. It indicates that the recovery within $300 \mu \mathrm{m}$ lateral to the dendritic damage border was almost complete.

reperfusion was initiated after $65.1 \pm 1.7 \mathrm{~min}$ of MCAO (Fig. $5 A, B)$. In animals in which the structure improved with reperfusion $(n=8)$, the recovered area defined by where the percentage of blebbed dendrites was not significantly different from the pre- occlusion control extended $\sim 300 \mu \mathrm{m}$ lateral of the structural border toward the MCAO stroke core (Fig. 5D). For an example of lateral dendrites (toward the MCA core region) that fail to recover with reperfusion, see Figure $5 \mathrm{~A}$. The partially recovered area (defined by where the percentage of blebbed dendrites was significantly different from both occlusion and the preocclusion control) extended from 300 to 600 $\mu \mathrm{m}$ lateral of the structural border (limited by the size of craniotomy window). Dendrites toward the lateral aspect of the craniotomy did not recover as well with reperfusion (Fig. 5D). For comparison of different animals, all distance measurements were made from the approximate border of structural damage during MCAO (Fig. 5D). Recovery of structure began gradually after $\sim 20-30$ min of reperfusion and in some cases resulted in the appearance of dendrites that were nearly identical with prestroke conditions (Fig. $5 B, C$ ). In all eight animals that were reperfused and showed recovery, the recovered dendritic structure was maintained for at least $3 \mathrm{~h}$ after the beginning of the initial ischemic period.

\section{Irreversible structural damage within} the ischemic core

A potential limitation of two-photon microscopy was that it was difficult to simultaneously position both laser Doppler probes and two-photon imaging windows over the most lateral aspects of the cortex. In addition, curvature of the lateral cortex made it difficult to create a cranial window that permitted both the medial penumbra and the lateral ischemic core to be imaged using two-photon microscopy. Therefore, we used a histological analysis to establish whether core regions of the MCAOinduced stroke (including the more lateral cortical region and the striatum) would also recover their dendritic structure during confirmed reperfusion. After reperfusion, the ischemic penumbra exhibited recovered dendritic structure that we observed during live imaging. In contrast, after reperfusion we did not observe intact structure within core regions by histological analysis in all animals $(n=5)$ that showed recovery in the penumbra area (by live imaging) consistent with our previous histological work (Enright et al., 2007) (Fig. 6; supplemental Fig. 2, available at www.jneurosci.org as supplemental material). In our previous study in the core region (produced by irreversible MCAO), we showed that widespread loss of spines occurred in addition to blebbing of dendrites (Enright et al., 2007). Here, we find that dendrites in these cortical regions that were on average $>3.8 \pm 0.3 \mathrm{~mm}$ lateral from 
midline were still blebbed after reperfusion (Fig. $6 B, D, G$ ). This distance from the midline was $\sim 0.7 \mathrm{~mm}$ lateral to the dendritic structural border observed during live two-photon imaging experiments. In contrast to the MCAO core, histological analysis indicated that dendrites in more medial regions were in part recovered (Fig. $6 B, C$ ) (for group data, see supplemental Fig. 2, available at www.jneurosci.org as supplemental material) and those in the contralateral hemisphere remained intact (Fig. 6A). Axons within the striatum of the ischemic hemisphere were also beaded, but not in the contralateral hemisphere (Fig. 6E,F).

\section{Discussion}

Stroke can have devastating consequences for neuronal structure, function, and survival. We have shown previously in vivo that dendrites can recover from $7 \mathrm{~min}$ of global ischemia (Murphy et al., 2008), as well as brief interruption in local blood flow triggered by photothrombotic infarction (Zhang et al., 2005). Limitations of previous studies were that they did not produce defined reductions in blood flow that approached the spatial patterns and durations observed during human stroke (Siesjo, 1992a,b). Here, we adapt twophoton imaging to accommodate occlusion of the MCA in mice with fluorescent cortical neurons. We show that the dendritic arbors of these neurons within the penumbra can recover after even $60 \mathrm{~min}$ of sustained ischemia with blood flow at levels $<20 \%$ of normal values.

How much brain can be saved and how long after occlusion?

An obvious question is how long occlusion can be maintained before dendritic structure fails to recover with reperfusion? One would assume that, if human patients can undergo significant restoration of brain function after thrombolysis after up to $3 \mathrm{~h}$ of occlusion, it would also be possible within the mouse to observe recovered dendrites after these occlusion times.

However, we caution that reperfusion at 90 min or longer occlusion in mice may be complicated by incomplete or variable reperfusion (our unpublished data). Thus, it is conceivable that precise translation between rodent vulnerability and humans may be difficult to make. Possibly, different metabolic rates of mice and humans may make mice more susceptible to relatively shorter durations of ischemia. It is also conceivable that, in cases of human stroke, other sources of blood flow may be available through collaterals or unaffected arterial systems allowing tissues to be maintained at relatively higher oxygen tension permitting recovery of synaptic structure with longer reperfusion times. Hopefully, these and other factors extend the window for reperfusion to restore dendrite and synapse structure in stroke patients. How- ever, it should be emphasized that the reperfusion time window for human stroke using thrombolysis is most effective if patients were treated within 90 min after the onset of symptoms (Mehdiratta and Caplan, 2007) resembling the time points we study.

Another major question concerns the size of the recoverable penumbra. Using the MCAO model, we find dendritic structure that is maintained in a relatively intact state up to a millimeter within partially ischemic areas (Fig. $7 A, B$ ). It is conceivable that these structurally intact tissues will be nonfunctional during occlusion, but will regain function during reperfusion. In previous work, we show that individual blood vessels can support the structure of dendrites that were on average only $80 \mu \mathrm{m}$ into an ischemic zone (Zhang and Murphy, 2007). We should note some 


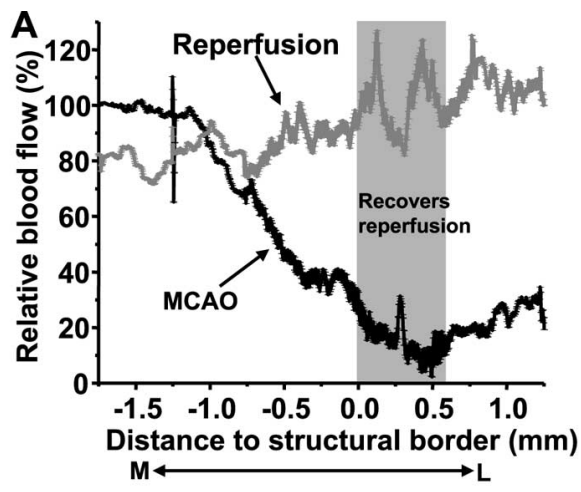

B

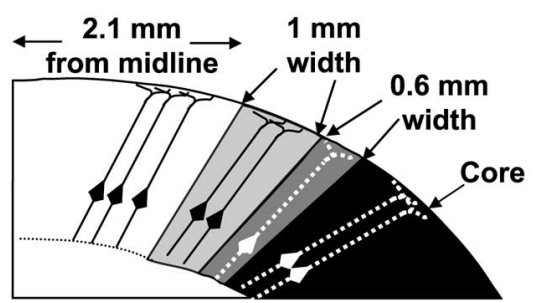

Reduced blood supply, relatively no structural damage during MCAO or reperfusion

Damaged structure, can recover during reperfusion

Damaged structure, no recovery during reperfusion

Figure 7. Summary diagram showing the relationship between the ischemic core and hypoperfused area including the penumbra after MCA0.A, Regional quantification of microcirculation (mean \pm SEM) using laser speckle within the two-photon imaging window (average data from 8 animals are shown). Data expressed as percentage of prestroke level (inverted and scaled) (see Materials and Methods) so that increasing values correspond to increasing blood flow. This analysis shows that the area up to $\sim 1 \mathrm{~mm}$ medial to the structural border was hypoperfused but relatively structurally intact. Blood flow was depressed to $<20 \%$ (during occlusion) within the area recovered after reperfusion (shaded area). Across most of the MCA territory, blood flow was restored to nearly $100 \%$ of prestroke levels after reperfusion. $\boldsymbol{B}$, The light gray area, which is $\sim 1 \mathrm{~mm}$ medial to the structural border, is where the blood flow was reduced (according to speckle laser data) and the dendritic structure remained mostly intact (according to two-photon microscopy data). The darker gray area, which is $\sim 0.6 \mathrm{~mm}$ lateral to the structural border, is where the dendrite tufts of the layer 5 pyramidal neurons blebbed during transient $M C A 0$, and recovered either fully or partially after reperfusion. The black area, which begins $\sim 3.7 \mathrm{~mm}$ lateral to midline (according to histological data), marks the more medial region of the MCAO core. Histological analysis indicated that dendritic structure in the core did not recover after reperfusion.

important differences between this previous work using photothrombosis to block local blood flow (Zhang and Murphy, 2007) versus occlusion of large arteries such as the MCA. Photothrombosis of surface arteries results in a very strong local reduction in blood flow (Watson et al., 1985; Schaffer et al., 2006), apparently affecting most blood vessels within the area, generating areas with little or no blood flow separated by a transition zone of only a few tens of micrometers in which blood flow begins again (Zhang and Murphy, 2007). The situation is quite different from strokes generated by MCAO in mice. In this model, we find an overlapping watershed area that is supplied by the lateral MCA, as well as the more medial ACA system. This leads to a relatively wide zone up to a millimeter across in which neurons are supplied by blood flow from both arterial systems. It is also conceivable that, in human patients, the angioarchitecture of the ACA/MCA watershed region will create a relatively larger penumbra than in the rodent.

Interestingly, the ability to recover dendritic structure with reperfusion appears to be unique to the ischemic penumbra in a 0.6-mm-wide region between MCA and ACA territories (Fig. $7 B$ ). The blood supply within this region during occlusion was $<20 \%$ of baseline levels. This suggests that potentially salvageable dendritic structure can be within a severely hypoperfused region, as long as it is close to a normally perfused area and reperfused in a timely manner. A limitation of our work was that reperfusion was more reliable if the suture was inserted through the CCA. In this case, we needed to permanently occlude the CCA, leaving animals only reperfused by a single CCA (reperfused to within $\sim 75 \%$ of prestroke blood flow).

\section{Irreversible loss of dendritic structure in core regions}

The more lateral stroke core would be expected to be more dependent on the MCA. Dendritic damage would be expected to be worse here because there would be a smaller contribution from unaffected blood supplies (such as the ACA system). We observe this to be correct and found that reperfusion was not as effective at normalizing dendritic structure as we moved to more lateral regions. With two-photon imaging, we observed that the most lateral areas of the craniotomy showed the lowest potential for recovery with reperfusion. To further check this in even more lateral areas, we did histological studies and observed that, in animals successfully reperfused for $1.5-3 \mathrm{~h}$, there were still blebbed dendrites within the MCA core region despite the observation (using two-photon imaging) that penumbra dendrites in part recovered (Fig. 6). Interestingly, blebbed dendritic structure was greatest near the brain surface consistent with a histological study we had done previously with permanent MCAO (Enright et al., 2007). Although we have not studied neuronal structure for $>3 \mathrm{~h}$ after reperfusion, it is possible that some neurons will undergo cell death over 24-48 h (Linnik et al., 1993; MacManus et al., 1993; Nitatori et al., 1995). Surviving neurons closer to the penumbra may lose synaptic inputs from their dendritic tuft within layer 1 cortex and rely on deeper more intact circuitry (Enright et al., 2007). The lack of recovered structure in core regions in our hands was not attributable to inadequate reperfusion. Although the ischemic core was typically outside our twophoton imaging window, we did have a laser Doppler probe present in this area and observed reperfusion (data not shown). Our work showing little recovery of core dendrites after 90 min of reperfusion has broad implications for neuroprotection studies because it would imply that, even if neurons could be protected from delayed cell death, their circuitry may already be irreversibly damaged.

\section{Reperfusion damage, stroke core, and penumbra}

Assessment of group data indicated that the reperfusion of neurons within the penumbra led to an improvement in dendritic structure, arguing against reperfusion damage in this area (Walder et al., 1997; Miller et al., 2006). However, it is conceivable that the MCA core, which is inaccessible to two-photon imaging (because it was more lateral and curved), could have undergone reperfusion damage or that somehow reactive oxygen species present during reperfusion would prevent recovery of dendritic structure (Winquist and Kerr, 1997; Jean et al., 1998; del Zoppo et al., 2000). Future work using larger craniotomies that cover the more lateral aspect of the brain would permit imaging of structure directly within the MCA core of live animals.

Implications for animal models of stroke neuroprotection Our study clearly differentiates tissues that can recover during reperfusion from those that cannot. We feel the work will help guide neuroprotection assays because two-photon imaging can more accurately measure the stroke penumbra. Although we 
show that structure fails to recover within the MCA core with reperfusion, we do not rule out that long-term neuroprotection within these sites is possible. Conceivably, even if cell death cascades were initiated (after 60 min of occlusion), neurons could be treated with an antinecrotic or antiapoptotic agent after reperfusion. Although neurons within the core could be made to survive, they may have compromised synaptic transmission because we would not expect structural recovery of their apical dendritic arbors. Recent work indicates that this may be the case because antiapoptotic CDK-5 inhibitors block cell death but fail to fully restore synaptic transmission and behavior after stroke (Iyirhiaro et al., 2008).

A limitation is that we do not image for $>3 \mathrm{~h}$ after reperfusion; however, we would argue that, if the ischemic core synaptic structure cannot be recovered at $3 \mathrm{~h}$, it is unlikely that it would be recovered later. Within the penumbra (in which dendritic structure can recover with reperfusion), we do not know whether some of these neurons may be subject to cell death pathways or loss of dendrites at time points $>3-4 \mathrm{~h}$ after stroke onset. Regardless of the long-term outcome, reperfusion-induced recovery of structure within the penumbra is still relevant. Possibly, agents or treatments (Stenzel-Poore et al., 2007) that reduce later cell death may ensure that penumbra neurons with recovered structure during reperfusion survive and contribute to synaptic networks. In conclusion, we suggest that neuroprotection studies not only focus on saving neuronal cell bodies from death but also gear treatments to retain or regenerate synaptic structure to ensure that both the neuron and its circuitry survive stroke.

\section{References}

Andrew RD, Labron MW, Boehnke SE, Carnduff L, Kirov SA (2007) Physiological evidence that pyramidal neurons lack functional water channels. Cereb Cortex 17:787-802.

Aronowski J, Strong R, Grotta JC (1997) Reperfusion injury: demonstration of brain damage produced by reperfusion after transient focal ischemia in rats. J Cereb Blood Flow Metab 17:1048-1056.

Bethel A, Kirsch JR, Koehler RC, Finklestein SP, Traystman RJ (1997) Intravenous basic fibroblast growth factor decreases brain injury resulting from focal ischemia in cats. Stroke 28:609-615; discussion 615-616.

Briers JD, Fercher AF (1982) Retinal blood-flow visualization by means of laser speckle photography. Invest Ophthalmol Vis Sci 22:255-259.

Chen H, Chopp M, Zhang ZG, Garcia JH (1992) The effect of hypothermia on transient middle cerebral artery occlusion in the rat. J Cereb Blood Flow Metab 12:621-628.

Chen JF, Huang Z, Ma J, Zhu J, Moratalla R, Standaert D, Moskowitz MA, Fink JS, Schwarzschild MA (1999) $\mathrm{A}_{2 \mathrm{~A}}$ adenosine receptor deficiency attenuates brain injury induced by transient focal ischemia in mice. J Neurosci 19:9192-9200.

del Zoppo G, Ginis I, Hallenbeck JM, Iadecola C, Wang X, Feuerstein GZ (2000) Inflammation and stroke: putative role for cytokines, adhesion molecules and iNOS in brain response to ischemia. Brain Pathol 10:95-112.

Dunn AK, Bolay H, Moskowitz MA, Boas DA (2001) Dynamic imaging of cerebral blood flow using laser speckle. J Cereb Blood Flow Metab 21:195-201.

Durukan A, Tatlisumak T (2007) Acute ischemic stroke: overview of major experimental rodent models, pathophysiology, and therapy of focal cerebral ischemia. Pharmacol Biochem Behav 87:179-197.

Enright LE, Zhang S, Murphy TH (2007) Fine mapping of the spatial relationship between acute ischemia and dendritic structure indicates selective vulnerability of layer $\mathrm{V}$ neuron dendritic tufts within single neurons in vivo. J Cereb Blood Flow Metab 27:1185-1200.

Feng G, Mellor RH, Bernstein M, Keller-Peck C, Nguyen QT, Wallace M, Nerbonne JM, Lichtman JW, Sanes JR (2000) Imaging neuronal subsets in transgenic mice expressing multiple spectral variants of GFP. Neuron 28:41-51.

Gürsoy-Ozdemir Y, Can A, Dalkara T (2004) Reperfusion-induced oxida- tive/nitrative injury to neurovascular unit after focal cerebral ischemia. Stroke 35:1449-1453.

Hara H, Huang PL, Panahian N, Fishman MC, Moskowitz MA (1996) Reduced brain edema and infarction volume in mice lacking the neuronal isoform of nitric oxide synthase after transient MCA occlusion. J Cereb Blood Flow Metab 16:605-611.

Hori N, Carpenter DO (1994) Functional and morphological changes induced by transient in vivo ischemia. Exp Neurol 129:279-289.

Hossmann KA (1996) Periinfarct depolarizations. Cerebrovasc Brain Metab Rev 8:195-208.

Hossmann KA (2006) Pathophysiology and therapy of experimental stroke. Cell Mol Neurobiol 26:1057-1083.

Iijima T, Mies G, Hossmann KA (1992) Repeated negative DC deflections in rat cortex following middle cerebral artery occlusion are abolished by MK-801: effect on volume of ischemic injury. J Cereb Blood Flow Metab 12:727-733.

Iyirhiaro GO, Brust TB, Rashidian J, Galehdar Z, Osman A, Phillips M, Slack RS, Macvicar BA, Park DS (2008) Delayed combinatorial treatment with flavopiridol and minocycline provides longer term protection for neuronal soma but not dendrites following global ischemia. J Neurochem 105:703-713.

Jean WC, Spellman SR, Nussbaum ES, Low WC (1998) Reperfusion injury after focal cerebral ischemia: the role of inflammation and the therapeutic horizon. Neurosurgery 43:1382-1396; discussion 1396-1397.

Kamii H, Kinouchi H, Sharp FR, Koistinaho J, Epstein CJ, Chan PH (1994) Prolonged expression of hsp70 mRNA following transient focal cerebral ischemia in transgenic mice overexpressing $\mathrm{CuZn}$-superoxide dismutase. J Cereb Blood Flow Metab 14:478-486.

Kawamura S, Yasui N, Shirasawa M, Fukasawa H (1991) Rat middle cerebral artery occlusion using an intraluminal thread technique. Acta Neurochir (Wien) 109:126-132.

Kleinfeld D, Mitra PP, Helmchen F, Denk W (1998) Fluctuations and stimulus-induced changes in blood flow observed in individual capillaries in layers 2 through 4 of rat neocortex. Proc Natl Acad Sci U S A 95:15741-15746.

Linnik MD, Zobrist RH, Hatfield MD (1993) Evidence supporting a role for programmed cell death in focal cerebral ischemia in rats. Stroke 24:20022008; discussion 2008-2009.

Longa EZ, Weinstein PR, Carlson S, Cummins R (1989) Reversible middle cerebral artery occlusion without craniectomy in rats. Stroke 20:84-91.

Ma J, Endres M, Moskowitz MA (1998) Synergistic effects of caspase inhibitors and MK-801 in brain injury after transient focal cerebral ischaemia in mice. Br J Pharmacol 124:756-762.

MacManus JP, Buchan AM, Hill IE, Rasquinha I, Preston E (1993) Global ischemia can cause DNA fragmentation indicative of apoptosis in rat brain. Neurosci Lett 164:89-92.

Mehdiratta M, Caplan LR (2007) Acute [corrected] stroke thrombolysis: an update [corrected]. Prog Cardiovasc Dis 49:430-438.

Miller AA, Dusting GJ, Roulston CL, Sobey CG (2006) NADPH-oxidase activity is elevated in penumbral and non-ischemic cerebral arteries following stroke. Brain Res 1111:111-116.

Murphy TH, Li P, Betts K, Liu R (2008) Two-photon imaging of stroke onset in vivo reveals that NMDA-receptor independent ischemic depolarization is the major cause of rapid reversible damage to dendrites and spines. J Neurosci 28:1756-1772.

Nishimura N, Schaffer CB, Friedman B, Tsai PS, Lyden PD, Kleinfeld D (2006) Targeted insult to subsurface cortical blood vessels using ultrashort laser pulses: three models of stroke. Nat Methods 3:99-108.

Nitatori T, Sato N, Waguri S, Karasawa Y, Araki H, Shibanai K, Kominami E, Uchiyama Y (1995) Delayed neuronal death in the CA1 pyramidal cell layer of the gerbil hippocampus following transient ischemia is apoptosis. J Neurosci 15:1001-1011.

Park JS, Bateman MC, Goldberg MP (1996) Rapid alterations in dendrite morphology during sublethal hypoxia or glutamate receptor activation. Neurobiol Dis 3:215-227.

Schaffer CB, Friedman B, Nishimura N, Schroeder LF, Tsai PS, Ebner FF, Lyden PD, Kleinfeld D (2006) Two-photon imaging of cortical surface microvessels reveals a robust redistribution in blood flow after vascular occlusion. PLoS Biol 4:e22.

Siesjo BK (1992a) Pathophysiology and treatment of focal cerebral ischemia. Part I: Pathophysiology. J Neurosurg 77:169-184.

Siesjo BK (1992b) Pathophysiology and treatment of focal cerebral isch- 
emia. Part II: Mechanisms of damage and treatment. J Neurosurg 77:337-354

Sigler A, Goroshkov A, Murphy TH (2008) Hardware and methodology for targeting single brain arterioles for photothrombotic stroke on an upright microscope. J Neurosci Methods 170:35-44.

Stenzel-Poore MP, Stevens SL, King JS, Simon RP (2007) Preconditioning reprograms the response to ischemic injury and primes the emergence of unique endogenous neuroprotective phenotypes: a speculative synthesis. Stroke 38:680-685.

Suzuki Y, Masuda K, Ogino K, Sugita T, Aizu Y, Asakura T (1991) Measurement of blood flow velocity in retinal vessels utilizing laser speckle phenomenon. Jpn J Ophthalmol 35:4-15.

Walder CE, Green SP, Darbonne WC, Mathias J, Rae J, Dinauer MC, Curnutte JT, Thomas GR (1997) Ischemic stroke injury is reduced in mice lacking a functional NADPH oxidase. Stroke 28:2252-2258.
Watson BD, Dietrich WD, Busto R, Wachtel MS, Ginsberg MD (1985) Induction of reproducible brain infarction by photochemically initiated thrombosis. Ann Neurol 17:497-504.

Winquist RJ, Kerr S (1997) Cerebral ischemia-reperfusion injury and adhesion. Neurology 49:S23-S26.

Yuan S, Devor A, Boas DA, Dunn AK (2005) Determination of optimal exposure time for imaging of blood flow changes with laser speckle contrast imaging. Appl Opt 44:1823-1830.

Zhang S, Murphy TH (2007) Imaging the impact of cortical microcirculation on synaptic structure and sensory-evoked hemodynamic responses in vivo. PLoS Biol 5:e119.

Zhang S, Boyd J, Delaney K, Murphy TH (2005) Rapid reversible changes in dendritic spine structure in vivo gated by the degree of ischemia. J Neurosci 25:5333-5338. 